\title{
Development of Planar Schottky Diode on GaAs Substrate for Terahertz Applications
}

\author{
Won-Young Uhm*, Seok-Gyu Choi*, Min Han*, Keun-Kwan Ryu** and Sung-Chan Kim ${ }^{\dagger}$
}

\begin{abstract}
In this paper, we demonstrate the planar Schottky diode on GaAs substrate for terahertz applications. A nanoscale dot and T-shaped disk has been developed as the anode for terahertz Schottky diode. The low parasitic elements of the nanoscale anode with T-shaped disk yield high cutoff frequency characteristic. The fabricated Schottky diode with anode diameter of $500 \mathrm{~nm}$ has series resistance of $21 \Omega$, ideality factor of 1.32 , junction capacitance of $8.03 \mathrm{fF}$, and cutoff frequency of $944 \mathrm{GHz}$.
\end{abstract}

Keywords: Schottky diode, Nanoscale dot, Terahertz, Anode, GaAs

\section{Introduction}

In the recent years there has been an increasing demand for higher frequencies in various fields because most available microwave bands are already exhausted. This demand is anticipated to further increase to approach the terahertz frequency range $(100 \mathrm{GHz}$ to $10 \mathrm{THz})$ in the near future. The terahertz frequency range has a lot of potential applications such as imaging system, short range radar system, high speed data communication system, and so on [1].

In order to realize various terahertz applications, the high performance semiconductor device is very important. One of the promising candidates for semiconductor device is the Schottky diode. The Schottky diode is a majoritycarrier device that does not suffer from charge-storage effects, and therefore it provides good and uniform electrical characteristics [2-3]. Furthermore fabrication process for Schottky diode is comparatively easy and, at the same time, allows the implementation of other devices on the same substrate.

Most diodes for high frequency applications use a circular anode with an air-bridge that connects the anode to the ohmic contact. As the anode diameter is reduced for higher frequency operation, a series resistance increases and a width of the air-bridge becomes larger than the anode diameter. This structure induces strong parasitic elements and results in deteriorating the device performance. It also causes a physical instability for the device fabrication.

In this paper we report on the development of the planar Schottky diode with nanoscale anode and T-shaped disk on GaAs substrate for terahertz applications. This structure results in lower parasitic elements compared with

$\dagger$ Corresponding Author: Dept. of Electronics and Control Engineering, Hanbat National University, Korea. (sckim@hanbat.ac.kr)

* Division of Electronics and Electrical Engineering, Dongguk University, Korea.

** Dept. of Electronics and Control Engineering, Hanbat National University, Korea.

Received: February 24, 2016; Accepted: April 18, 2016 conventional air-bridge type diodes.

\section{Schottky Diode Design and Fabrication}

There are three design parameters that can be used to optimize the Schottky diode. These are the anode diameter, the epitaxial layer thickness, and the epitaxial layer doping density. As frequency is increased, anode diameter must decrease and the epitaxial doping density must increase. The epitaxial layer thickness should be no greater than a zero-bias depletion depth in epitaxial layer.

Schottky diode is the critical element used in various circuits for a variety of applications in the sub-millimeter and terahertz frequency range. For high performance characteristics of the circuit, the operating frequency of the circuit should be significantly lower than diode's cutoff frequency. The diode cutoff frequency is often defined as Eq. (1).

$$
f_{c}=\frac{1}{2 \pi R_{s}\left(C_{j 0}+C_{p}\right)}
$$

$R_{s}$ is the DC measured series resistance at large forward bias. $C_{p}$ is the parasitic capacitance and $C_{j 0}$ is the junction capacitance at zero-bias. This expression ignores the variation of the parasitic capacitance, the junction capacitance with bias, and the high-frequency components of the series impedance. However its main benefits are that it is easily measured and the diodes with the greatest $f_{c}$ virtually always yield the best performance at very high frequencies. Reducing the anode diameter reduces junction capacitance, but increases the series resistance. Thus, complicated trade-offs are involved in choosing an anode size. However, some research has shown that reducing the anode diameter leads to better performance at high frequency [4]. Similar analysis using the $R_{s} C_{j 0}$ product indicates that the optimum anode diameter maximizes the 
cutoff frequency for each epitaxial layer doping density [5]. Therefore we calculated the series resistance and the junction capacitance as a function of the anode diameters. From the simulated results, we concluded that the suitable anode diameter was $500 \mathrm{~nm}$ considering optimized $R_{s}$ and $C_{j 0}$ as well as process stability.

The thickness of the epitaxial layer causes an increase in series impedance, but it leads to a decrease in the junction capacitance if the depletion region extends through the entire layer. The maximum thickness of the depletion layer of the Schottky diode with DC bias is dominated by the thickness of the epitaxial layer. Reducing the thickness of the epitaxial layer causes an increase in the diode capacitance and a reduction of the diode series resistance. The trade-off between these two effects needs to be considered in order to determine the optimum thickness of the epitaxial layer [6].

Epitaxial structures for the Schottky diodes were grown on 4-inch semi-insulating GaAs substrate by using molecular beam epitaxy (MBE). GaAs buffer layers with a thickness of $300 \mathrm{~nm}$ were grown on the GaAs substrate. Ohmic layers were grown with a thickness of $300 \mathrm{~nm}$ with Si doping $\left(6 \times 10^{18} / \mathrm{cm}^{3}\right)$ for the ohmic contact. The top of the epitaxial layer was grown with Si doping $\left(1 \times 10^{17} / \mathrm{cm}^{3}\right)$ for the Schottky contact. The doping density has an important effect on the diode characteristics such as junction capacitance, series impedance, and sharpness of the I-V curve. Increasing the Si doping density causes a reduction of the series resistance, but also leads to a steep increase in the ideality factor $(\eta)$. A simple analysis indicates that cutoff frequency is increased by increasing the epitaxial layer doping density.

The Schottky diode was fabricated using the standard MMIC process of MINT, Dongguk University [7]. MINT MMIC process steps include mesa isolation, evaporation of ohmic metal, resistor, first metal, deposition of $\mathrm{Si}_{3} \mathrm{~N}_{4}$, airbride formation and so on. The anode of the Schottky diode was fabricated using heterogeneous resist patterning method. A photo lithography process is unsuitable for nanoscale patterning. Therefore we performed nano-dot patterning by e-beam lithography process. The nanoscale dot as the anode is used for the Schottky contact and the T-

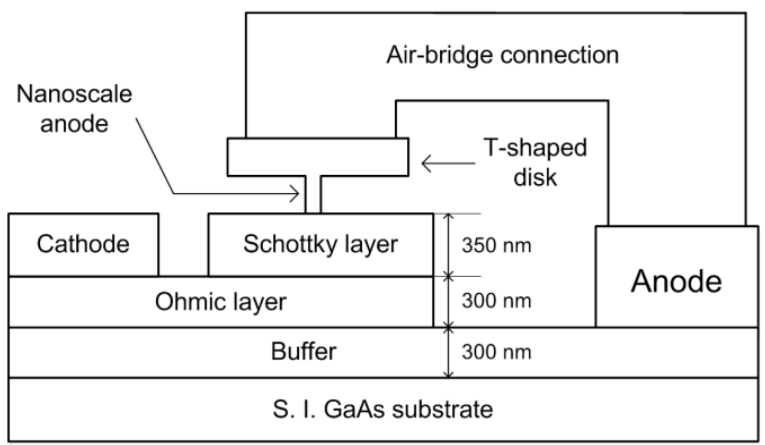

Fig. 1. Schematic diagram of the developed Schottky diode with nanoscale anode (not to scale) shaped disk is used to connect the anode to air-bridge. The e-beam lithography process uses multiple e-beam scans at different doses and a tri-level e-beam resist system that consists of PMMA950K and PMGI in order to separately pattern the nanoscale dot and the T-shaped disk of the

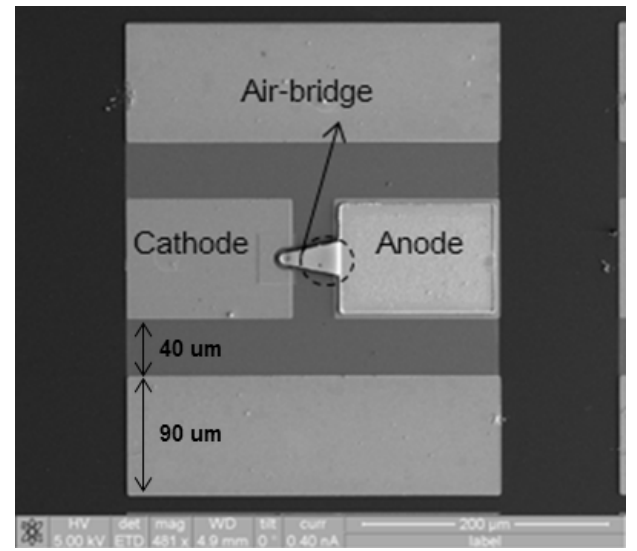

Fig. 2. SEM photograph of the fabricated Schottky diode: top view

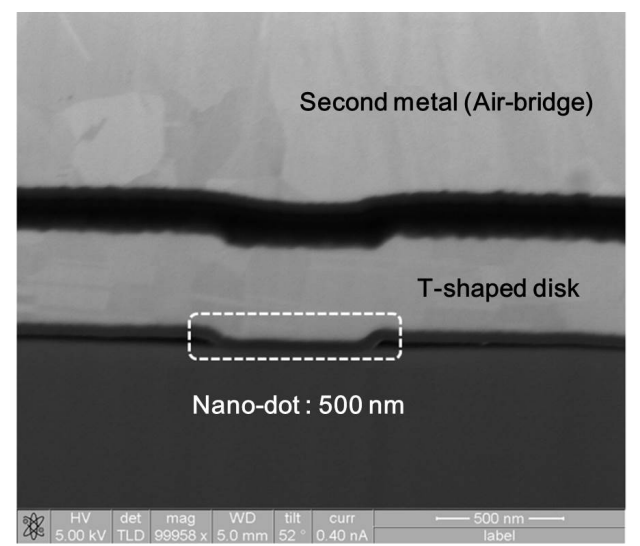

Fig. 3. SEM photograph of the fabricated Schottky diode: nanoscale anode (diameter: $500 \mathrm{~nm}$ )

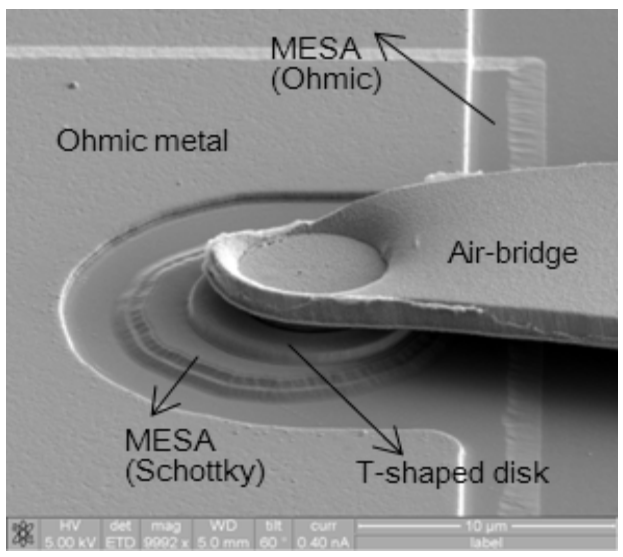

Fig. 4. SEM photograph of the fabricated Schottky diode: close-up of the cathode area 
diode anode. After metallization for diode anode, air-bridge process was performed by photo lithography in order to connect anode with anode pad.

Fig. 1 and Fig. 2 show a schematic diagram and a SEM photograph of the fabricated Schottky diode with nano-dot structure, respectively.

Fig. 3 shows a nanoscale anode and Fig. 4 shows a close-up of the fabricated nanoscale dot and T-shaped disk.

\section{Characteristics of the Schottky Diode}

The I-V characteristics of the fabricated Schottky diode were measured using a Keithley 4200-SCS (semiconductor characterization system). The ideality factor $(\eta)$, current parameter $\left(I_{0}\right)$, and series resistance $\left(R_{s}\right)$ can be obtained from the measurement of the I-V characteristics.

Fig. 5 shows the I-V characteristics of the fabricated Schottky diodes. The extraction of the series resistance of the Schottky diode can be done by the saturation current method. The ideality factor is also obtained by calculating

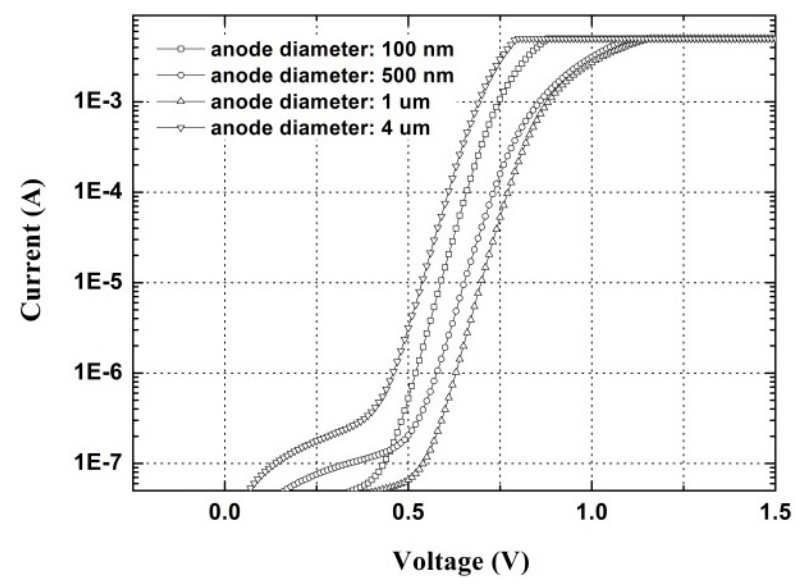

Fig. 5. Measured I-V characteristics of the fabricated Schottky diodes

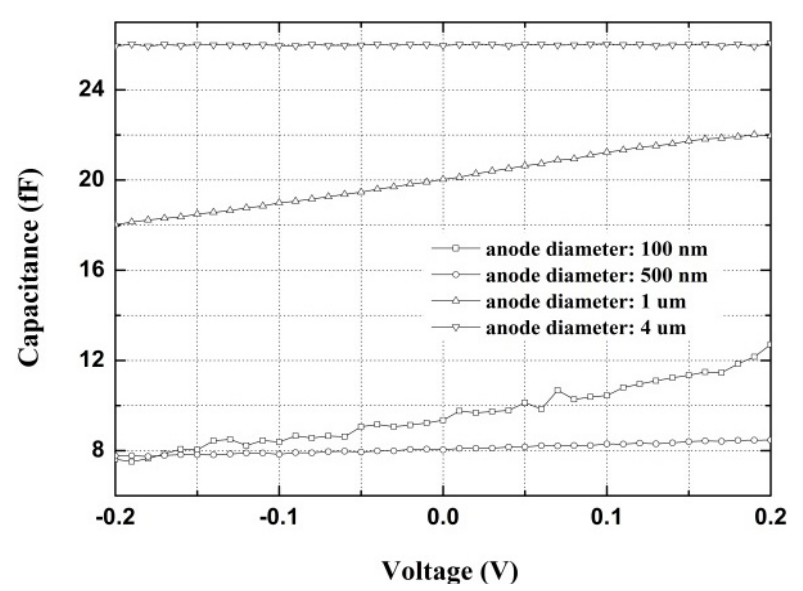

Fig. 6. Measured total capacitance characteristics of the fabricated Schottky diodes a voltage deviation between fitting line and measured I-V curve.

The capacitance-voltage characteristics are measured using an Agilent E4980A Precision LCR Meter. Fig. 6 shows the measured total capacitance characteristics. The total capacitance consists of the junction capacitance $\left(C_{j 0}\right)$ and the parasitic capacitance $\left(C_{p}\right)$. Cutoff frequency can be calculated in Eq. (1) using the obtained series resistance and the total capacitance.

The S-parameters characteristics are measured using the Agilent $8510 \mathrm{C}$ vector network analyzer. Fig. 7 shows the measured S-parameters characteristics of the fabricated Schottky diode with anode diameter of $500 \mathrm{~nm}$ in the frequency range from $1 \mathrm{GHz}$ to $50 \mathrm{GHz}$.

Measured DC characteristics and cutoff frequency of the fabricated Schottky diode are given in Table 1. The cutoff

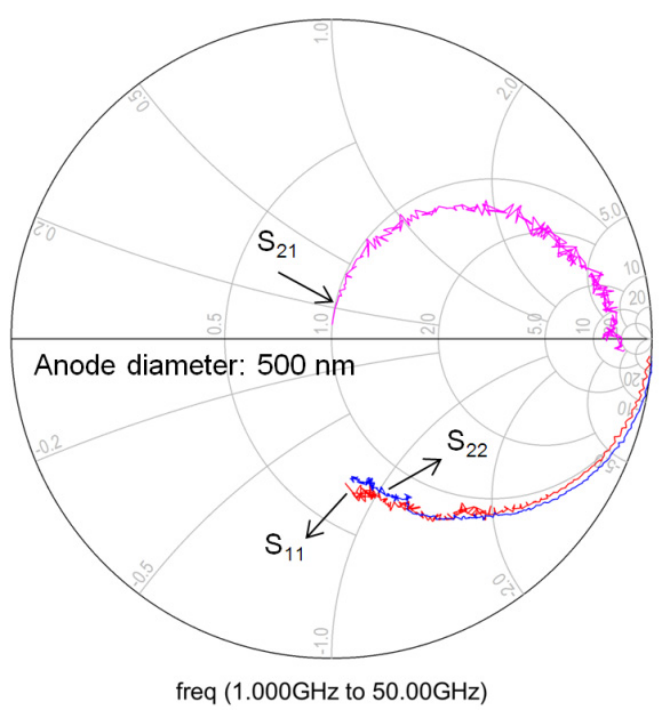

Fig. 7. Measured S-parameters characteristics of the fabricated Schottky diode from $1 \mathrm{GHz}$ to $50 \mathrm{GHz}$

Table 1. Measured results of the fabricated Schottky diodes

\begin{tabular}{c|c|c|c|c}
\hline Anode diameter & $\eta$ & $C_{\text {total }}(\mathrm{fF})$ & $R_{s}(\Omega)$ & $f_{c}(\mathrm{GHz})$ \\
\hline $100 \mathrm{~nm}$ & 1.18 & 9.35 & 20.6 & 826 \\
\hline $500 \mathrm{~nm}$ & 1.32 & 8.03 & 21 & 944 \\
\hline $1 \mu \mathrm{m}$ & 1.22 & 20 & 14.9 & 589 \\
\hline $4 \mu \mathrm{m}$ & 1.25 & 25.96 & 11.2 & 547 \\
\hline
\end{tabular}

Table 2. Comparison of cutoff frequency for the Schottky diode with the other published data

\begin{tabular}{c|c|c}
\hline Process technology & $f_{c}(\mathrm{GHz})$ & Ref. \\
\hline InGaAs HEMT & 370 & {$[8]$} \\
\hline GaAs MHEMT & 194 & {$[9]$} \\
\hline InGaAs HEMT & 908 & {$[10]$} \\
\hline GaAs MBE Schottky & 1000 & {$[11]$} \\
\hline GaAs MBE Schottky & 650 & {$[12]$} \\
\hline Si-based CMOS & 400 & {$[13]$} \\
\hline GaAs HBT & 1020 & {$[14]$} \\
\hline GaAs MBE Schottky & 944 & This work \\
\hline
\end{tabular}


frequency of the fabricated Schottky diode with anode diameter of $500 \mathrm{~nm}$ is $944 \mathrm{GHz}$, while those of the Schottky diode with anode diameter of $100 \mathrm{~nm}$ is $826 \mathrm{GHz}$. It seems that there are unexpected parasitic components due to process instability and measurement errors in the capacitance of the $100 \mathrm{~nm}$ pattern.

Table 2 shows a comparison of cutoff frequency performance for the fabricated Schottky diode with the other published data. These values indicate that the fabricated Schottky diode in this paper can be applied for the $94 \mathrm{GHz}$ detectors, $220 \mathrm{GHz}$ receivers, and the other terahertz applications in respect that the operating frequency is only about a third of cutoff frequency.

\section{Conclusion}

In this paper, we demonstrated the planar Schottky diode on GaAs substrate for terahertz applications. A nano-dot and T-shaped disk has been developed as the anode for terahertz Schottky diode. The low parasitic elements of the nanoscale anode with T-shaped disk yield high cutoff frequency characteristics. The fabricated Schottky diode with an anode diameter of $500 \mathrm{~nm}$ has series resistance of $21 \Omega$, ideality factor of 1.32 , junction capacitance of 8.03 $\mathrm{fF}$, and cutoff frequency of $944 \mathrm{GHz}$.

\section{Acknowledgements}

The authors would like to thank Millimeter-wave innovation research center (MINT), Dongguk University, Korea, for the circuit fabrication as well as Prof. Jin-Koo Rhee (MINT) for helpful discussion and characterization support.

\section{References}

[1] P. H. Siegel, "Terahertz technology," IEEE Trans. Microw. Theory Tech., 50(3), pp. 910-928, 2002.

[2] B. L. Sharma, Metal-semiconductor Schottky barrier junctions and their applications, Plenum, 1984.

[3] M. Shur, Physics of semiconductor devices, Prentice Hall, 1990.

[4] U. V. Bhapkar, and T. W. Crowe, "Analysis of the high frequency series impedance of GaAs Schottky diodes by a finite difference technique," IEEE Trans. Microw. Theory Tech., 40(5), pp. 886-894, 1992.

[5] T. W. Crowe, W. C. B. Peatman, P. A. D. Wood, and L. Xizolei, "GaAs Schottky barrier diodes for $\mathrm{THz}$ applications," in Proc. Proc. IEEE MTT-S. Int. Symp. Dig., pp. 1141-1144, 1992.
[6] T. Nozokido, J. J. Chang, C. M. Mann, T. Suzuki, and K. Mizuno, "Optimization of a Schottky Barrier Mixer Diode in the Submillimeter Wave Region," in Proc. Journal of Infrared and Millimeter Waves (JIRMW), 15(11), pp. 1851-1864, 1994.

[7] K. K. Ryu, D. An, J. K. Rhee, and S. C. Kim, "Highperformance CPW MMIC LNA Using GaAs-based Metamorphic HEMTs for 94-GHz Applications," $J$. Korean Phys. Soc., 56(5), pp. 1-6, 2010.

[8] K. W. Chang, H. Wang, T. H. Chen, K. Tan, G. S. Dow, A. C. Han, and L. T. Liu, "A W-band monolithic pseudomorphic InGaAs HEMT downconverter," in Proc. Microwave and Millimeter-wave Monolithic Circuits Symposium (MMWMC), pp. 55-58, 1991.

[9] M. Lee, B. Lim, S. Lee, D. Ko, S. Moon, D. An, H. Park, and J. Rhee, "A novel 94-GHz MHEMT-based diode mixer using a 3-dB tandem coupler," IEEE Microwave Wireless Component Letters, 18(9), pp. 626-628, 2008.

[10] S. H. Shin, D. Geum, and J. Jang, "MSM Varactor Diodes Based on $\mathrm{In}_{0.7} \mathrm{Ga}_{0.3}$ As HEMTs With Cut-Off Frequency of $908 \mathrm{GHz}$," IEEE Electron Dev. Lett., 35(2), pp. 172-174, 2014.

[11] J. Eisenberg, J. Panelli, and W. Ou, "A new planar double-double balanced MMIC mixer structure," in Proc. IEEE MTT-S. Int. Symp. Dig., pp. 81-84, 1991.

[12] J. Mou, Y. Yuan. X. Lv, W. Yu, D. He, J. Wang, and G. Xiao, "Design and fabrication of planar GaAs Schottky barrier diodes for submillimeter-wave applications," in Proc. IEEE Microwave and Millimeter Wave Technology (ICMMT), pp. 1746-1749, 2010.

[13] S. Sankarna, and K. K. O, "Schottky diode with cutoff frequency of $400 \mathrm{GHz}$ fabricated in $0.18 \mu \mathrm{m}$ CMOS," IEE Electronics Letters, 41(8), pp. 506-508, 2005.

[14] K. W. Kobayashi, R. Kasody, A. K. Oki, S. Dow, B. Allen, and D. C. Streit, "K-band double balanced mixer using GaAs HBT THz Schottky Diodes," in Proc. IEEE MTT-S. Int. Symp. Dig., pp. 1163-1166, 1994.

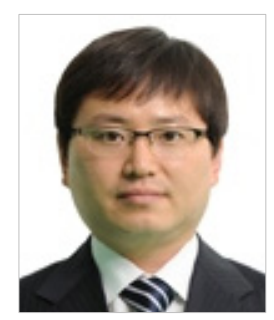

Won-Young Uhm He received his B.S. and M.S. in Electronic Engineering from Dongguk University, Seoul, Korea in 2002 and 2004, respectively. In 2004, he joined Agency for Defense Development (ADD) as a senior research engineer. He is currently pursuing his Ph.D. at Dongguk University. His research interests include high-frequency integrated circuits using compound semiconductor technologies 


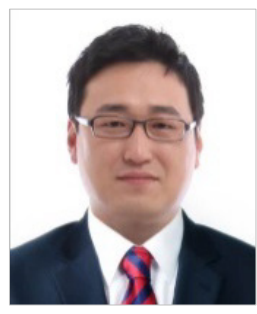

Seok-Gyu Choi He received his M.S. and Ph.D. degrees in Electronic Engineering from Dongguk University, Seoul, Korea, in 2006 and 2013, respectively. From 2013 to 2014, he was with the Millimeter-wave Innovation Technology (MINT) Research Center, Korea as a Post-doc. researcher. In 2014, he joined Gigalane as a senior researcher. His research interests include III-V and III-N compound semiconductor devices for RF applications.

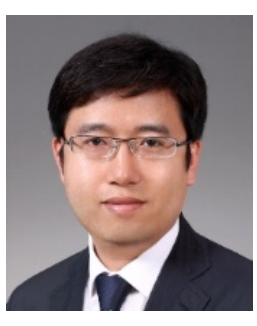

Min Han He received his M.S. and Ph.D. degrees in Electronic Engineering from Dongguk University, Seoul, Korea, in 2005 and 2012, respectively. From 2012 to 2013, he was with the Millimeter-wave Innovation Technology (MINT) Research Center, Korea as a Post-doc. researcher. In 2014, he joined RFHIC where he worked on the study of reliability of GaN HEMTs, GaAs MMIC and GaAs HBT.

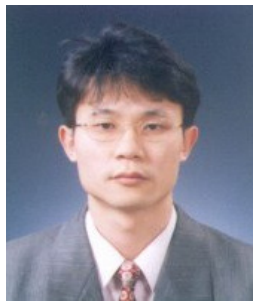

Keun-Kwan Ryu He received received his B.S., M.S., and Ph.D. in Electronics and Communications Engineering from Kwangwoon University, Seoul, Korea, in 1992, 1994, and 2000, respectively. From 2001 to 2002, he was with Electronics and Telecommunications Research Institute (ETRI) as a senior research engineer. In 2003, he joined Department of Electronic Engineering, Hanbat National University, Daejeon, Korea, and is now a full professor. His research interests include high-frequency active and passive circuits.

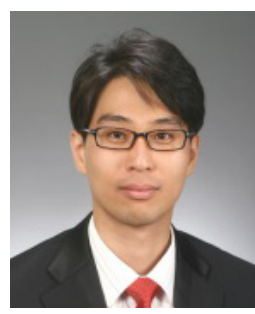

Sung-Chan Kim He received his B.S., M.S., and Ph.D. in Electronic Engineering from Dongguk University, Seoul, Korea, in 1999, 2001, and 2006, respectively. In 2007, he joined Department of Electronic Engineering, Hanbat National University, Daejeon, Korea, and is now an associate professor. His research interests include high-frequency integrated devices and circuits using compound semiconductor technologies at microwave and millimeter-wave frequencies. 